\title{
MEROMORPHIC FUNCTIONS THAT WEIGHTED SHARING THREE VALUES AND ONE PAIR
}

\author{
Thamir C. Alzahary and Hong-Xun Yi
}

\begin{abstract}
G. Brosch improved the theorem of Nevanlinna for four values theorem and proved that let $f$ and $g$ be two nonconstant meromorphic functions sharing $0,1, \infty \mathrm{CM}$, and let $a$ and $b$ be two finite complex numbers such that $a, b \notin\{0,1\}$. If $f=a \Leftrightarrow g=b$, then $f$ is a fractional linear transformation of $g$. In this paper we extend this theorem by using the idea of weighted sharing.
\end{abstract}

\section{Introduction, definitions and main results}

Let $f$ and $g$ be two nonconstant meromorphic functions on the open complex $\mathbf{C}$, and let $a$ be a finite value in the complex plane. We say that $f$ and $g$ share the value $a \mathrm{CM}$ (IM) provided that $f-a$ and $g-a$ have the same zeros counting multiplicities (ignoring multiplicities), and $f, g$ share $\infty \mathrm{CM}$ (IM) provided that $1 / f, 1 / g$ share $0 \mathrm{CM}$ (IM). Let $a, b \in \mathbf{C} \cup\{\infty\}$, if $f(z)=a$ when $g(z)=b$, then we denote it by $g(z)=b \Rightarrow f(z)=a$. We write $f(z)=a \Leftrightarrow g(z)$ $=b$ to mean that $f(z)=a$ if and only if $g(z)=b$. For standard notations and definitions of value distribution theory we refer [3].

We denote by $S(r, f)$ any function satisfying $S(r, f)=o(T(r, f))$ as $r \rightarrow+\infty$ possibly outside a set $E$ of finite Lebesgue measure. A meromorphic function $a(z)$ is said to be a small function of $f$, if $T(r, a)=S(r, f)$.

Throughout the paper we denote by $f, g$ two nonconstant meromorphic functions defined on the open complex plane and we use $N_{0}(r)$ to denote the counting function of the zeros of $f-g$ that are not zeros of $f(f-1), g(g-1)$, $\frac{1}{f}$ and $\frac{1}{g}$, unless the contrary is explicitly stated.

Definition 1 (see [6, p. 189]). Let $p$ be a positive integer, we denote by $N_{p)}(r, f)\left(\right.$ or $\left.\bar{N}_{p)}(r, f)\right)$ the counting function of poles of $f$ with multiplicities $\leq p$ (ignoring multiplicities). We further define

2000 Mathematics Subject Classification: 30D35, 30D30.

Key words and phrases: Meromorphic functions; Weighted sharing; Uniqueness.

Project Supported by the National Natural Science Foundation of China (No. 10371065).

Received January 27, 2005; revised June 13, 2005. 


$$
N_{(p+1}(r, f)=N(r, f)-N_{p)}(r, f), \quad \bar{N}_{(p+1}(r, f)=\bar{N}(r, f)-\bar{N}_{p)}(r, f) .
$$

R. Nevanlinna proved the following well-known theorem (see [6, p. 240]):

THeOREM A. Let $f$ and $g$ be two nonconstant meromorphic functions, and let $a_{j}(j=1,2,3,4)$ be four distanct shared values $C M$ by $f$ and $g$. Then $f$ is $a$ Möbius transformation (fractional linear transformation) of $g$.

The condition " $f$ and $g$ share four values CM" has been weakened to " $f$ and $g$ share two values CM and two values IM" by Gundersen (see [6, p. 262]). The following example which was found by G. Gundersen [4] in 1979:

Example 1. Let $f=\frac{e^{z}+1}{\left(e^{z}-1\right)^{2}}, g=\frac{1}{8} \frac{\left(e^{z}+1\right)^{2}}{e^{z}-1}$. Then $f, g$ share $0,1, \infty$ IM and $f=-\frac{1}{8} \Leftrightarrow g=-\frac{1}{8}$. It easy to verify that $f$ is not any fractional linear transformation of $g$.

From Theorem A, it is reasonable to ask whether the conclusion of Theorem $A$ is still true if we relax the condition of sharing four values CM to sharing three values IM and another one CM. This conjecture appears to be quite difficult. In 1989, Brosch [2] proved the following which is an improvement of Theorem A:

THEOREM B. Let $f$ and $g$ be two nonconstant meromorphic functions sharing $0,1, \infty C M$, and let $a$ and $b$ be two finite complex numbers such that $a, b \notin$ $\{0,1\}$. If $f=a \Leftrightarrow g=b$, then $f$ is a fractional linear transformation of $g$.

Now one may ask the following question: Can one be extended Theorem B by relaxing the nature of sharing the values 0,1 and $\infty$ ?

We can answer this question in the negative by Example 1. In this paper we will investigate this problem by using the following definition, which is called weighted sharing.

Definition 2 (see $[1,5])$. Let $k$ be a nonnegative integer or infinity. For any $a \in \mathbf{C} \cup\{\infty\}$, we denote by $E_{k}(a, f)$ the set of all $a$-points of $f$, where an $a$ point of multiplicity $m$ is counted $m$ times if $m \leq k$ and $k+1$ times if $m>k$. If $E_{k}(a, f)=E_{k}(a, g)$, we say that $f, g$ share the value $a$ with weight $k$.

The definition implies that if $f, g$ share a value $a$ with weight $k$ then $z_{0}$ is a zero of $f-a$ with multiplicity $m(\leq k)$ if and only if it is a zero of $g-a$ with multiplicity $m(\leq k)$ and $z_{0}$ is a zero of $f-a$ with multiplicity $m(>k)$ if and only if it is a zero of $g-a$ with multiplicity $n(>k)$, where $m$ is not necessarily equal to $n$. 
We write $f, g$ share $(a, k)$ to mean that $f, g$ share the value $a$ with weight $k$. Clearly if $f, g$ share $(a, k)$, then $f, g$ share $(a, p)$ for all integer $p, 0 \leq p<k$. Also we note that $f, g$ share a value $a$ IM or CM if and only if $f, g$ share $(a, 0)$ or $(a, \infty)$ respectively.

Remark 1 (see [6, Theorem 2.18]). Let $f$ and $g$ be two meromorphic functions sharing $0,1, \infty \mathrm{IM}$, then $T(r, f) \leq 3 T(r, g)+S(r, f)$ and $T(r, g) \leq 3 T(r, f)+$ $S(r, g)$. This shows that $S(r, f)=S(r, g)$. So that, in this paper we use $S(r)$ to denote $S(r, f)$ and $S(r, g)$, unless otherwise stated.

In this paper we improve Theorem B and obtain the following theorem:

THEOREM 1. Let $f$ and $g$ be two nonconstant meromorphic functions sharing $\left(a_{1}, 1\right),\left(a_{2}, \infty\right)$ and $\left(a_{3}, \infty\right)$, where $\left\{a_{1}, a_{2}, a_{3}\right\}=\{0,1, \infty\}$, and let $a$ and $b$ be two finite complex numbers such that $a, b \notin\{0,1\}$. If $f=a \Leftrightarrow g=b$, then $f$ is $a$ fractional linear transformation of $g$. Moreover, $f$ and $g$ satisfy exactly one of the following relations:

(i) $f \equiv g$; (ii) $f . g \equiv 1 ; \quad$ (iii) $f \equiv \frac{a}{b} g$; (iv) $f+g \equiv 1 ; \quad$ (v) $f \equiv a g$; (vi) $f \equiv(1-a) g+a ; \quad$ (vii) $\quad f \equiv \frac{1-a}{1-b} g+\frac{b-a}{b-1} ; \quad$ (viii) $f \equiv \frac{a g}{a-1+g} ; \quad$ (ix) $f=$ $\frac{a(b-1) g}{(b-a) g+(a-1) b} ;(\mathrm{x}) f \equiv \frac{g}{g-1}$.

The cases (ii) and (v) may occur if $a b=1$, cases (iv) and (viii) may occur if $a+b=1$, case (vi) and (x) may occur if $a b=a+b$.

Example 2. Let $f=e^{2 \gamma}+e^{\gamma}+1, \quad g=e^{-2 \gamma}+e^{-\gamma}+1, \quad a=\frac{3}{4} \quad$ and $b=3$. Then it is easily verified that $f$ and $g$ share $0,1, \infty \mathrm{CM}$, and $f=a \Rightarrow g=b$, $g=b \nRightarrow f=a$, but $f$ is not any fractional linear transformation of $g$. This shows that the condition $f=a \Leftrightarrow g=b$ in Theorem 1 is necessary.

Remark 2. The condition $a, b \notin\{0,1\}$ in the theorem 1 is necessary, as shown by the following example:

Example 3. Let $f=\frac{e^{z}+1}{e^{2 z}+1}$ and $g=\frac{e^{-z}+1}{e^{-2 z}+1}$. Obviously $f$ and $g$ share $0,1, \infty \mathrm{CM}$ and $f$ is not any fractional linear transformation of $g$. We see that $N_{1)}(r, f)+S(r, f)=2 N_{1)}\left(r, \frac{1}{f}\right)+S(r, f)=2 N_{1)}\left(r, \frac{1}{f-1}\right)+S(r, f)=T(r, f)$.

\section{Some lemmas}

For the proof of our result we need the following lemmas: 
Lemma 2.1 (see [1, Theorem 3]). Let $f$ and $g$ be two distinct nonconstant meromorphic functions sharing $\left(a_{1}, 1\right),\left(a_{2}, \infty\right)$ and $\left(a_{3}, \infty\right)$, where $\left\{a_{1}, a_{2}, a_{3}\right\}=$ $\{0,1, \infty\}$. If $f$ is not a fractional linear transformation of $g$, then for any $a \in$ $\mathbf{C} \backslash\{0,1\}$

(i) $N_{0}\left(r, \frac{1}{f^{\prime}}\right)=\bar{N}_{0}\left(r, \frac{1}{f^{\prime}}\right)+S(r, f), \quad \bar{N}\left(r, \frac{1}{f^{\prime}}\right)=\bar{N}_{0}\left(r, \frac{1}{f^{\prime}}\right)+S(r, f)$, the same identities hold for $\mathrm{g}$;

(ii) $N_{(3}\left(r, \frac{1}{f-a}\right)=S(r, f), N_{(3}\left(r, \frac{1}{g-a}\right)=S(r, g)$;

(iii) $T(r, f)=\bar{N}\left(r, \frac{1}{g^{\prime}}\right)+N_{0}(r)+S(r, f), \quad T(r, g)=\bar{N}\left(r, \frac{1}{f^{\prime}}\right)+N_{0}(r)+$ $S(r, f), N_{0}(r)=\bar{N}_{0}(r)+S(r, f) ;$

(iv) $T(r, f)=N\left(r, \frac{1}{f-a}\right)+S(r, f), T(r, g)=N\left(r, \frac{1}{g-a}\right)+S(r, f)$;

(v) $T(r, f)+T(r, g)=\bar{N}\left(r, \frac{1}{f}\right)+\bar{N}\left(r, \frac{1}{f-1}\right)+\bar{N}(r, f)+N_{0}(r)+S(r, f)$;

(vi) $N\left(r, \frac{1}{f-g}\right)=\bar{N}\left(r, \frac{1}{f-g}\right)+S(r, f)$,

where $N_{0}\left(r, \frac{1}{f^{\prime}}\right)\left(\bar{N}_{0}\left(r, \frac{1}{f^{\prime}}\right)\right)$ denotes the counting function corresponding to the zeros of $f^{\prime}$ that are not zeros of $f$ and $f-1$ (ignoring multiplicities) and $\bar{N}_{0}(r)$ is the reduced form of $N_{0}(r)$.

LeMma 2.2. Let $f$ and $g$ be nonconstant meromorphic functions. Suppose that $f$ satisfies the Riccati differential equation

$$
f^{\prime}=a+b f+c f^{2},
$$

where $a, b$ and $c(\not \equiv 0)$ are meromorphic small functions of $g$. Furthermore, let $\rho(\not \equiv f)$ be a meromorphic small function of $g$.

(i) $m(r, f)=S(r, f)+S(r, g)$;
(ii) If $\rho$ is a solution of $(2.1)$, then $\bar{N}\left(r, \frac{1}{f-\rho}\right)=S(r, f)+S(r, g)$; (iii) If $\rho$ is not any solution of $(2.1)$, then $N\left(r, \frac{1}{f-\rho}\right)=T(r, f)+S(r, f)+$
$S(r, g)$;

(iv) If $a \neq 0$, then $m\left(r, \frac{1}{f}\right)=S(r, f)+S(r, g)$.

Proof. From the assumption of Lemma 2.2,

When $|f| \geq 1$, then we have

$$
f=\frac{1}{c} \frac{f^{\prime}}{f}-\frac{a}{c} \frac{1}{f}-\frac{b}{c} .
$$

$$
|f| \leq \frac{1}{|c|}\left|\frac{f^{\prime}}{f}\right|+\left|\frac{a}{c}\right|+\left|\frac{b}{c}\right|
$$


It is required to show that $m(r, f)=S(r, f)+S(r, g)$, by using the definition of $m(r, f)$. If $a \neq \equiv 0$, then from the last equation, we get $m\left(r, \frac{1}{f}\right)=m(r, f)+$
$S(r, f)+S(r, g)$, which gives (iv), by using (i).

Set $h=f-\rho$, then from (2.1), we have

$$
h^{\prime}=\lambda+(b+2 c \rho) h+c h^{2},
$$

where $\lambda=-\rho^{\prime}+a+c \rho^{2}+b \rho$.

If $\rho$ is not any solution of (2.1), then $\lambda \not \equiv 0$ and by utilizing (iv), we get

$$
m\left(r, \frac{1}{f-\rho}\right)=m\left(r, \frac{1}{h}\right)=S(r, h)+S(r, g)
$$

which gives (iii).

If $\rho$ is a solution of $(2.1)$, then $\lambda \equiv 0$, and hence $\frac{h^{\prime}}{h}=(b+2 c \rho)+c h$, this shows that $\bar{N}\left(r, \frac{1}{h}\right) \leq N(r, b+2 c p)+S(r, g)=S(r, g)$, which gives (ii). This proves Lemma 2.2.

Lemma 2.3 (see [6, p. 220]). Let $f$ and $g$ be two nonconstant meromorphic functions such that $\bar{N}\left(r, \frac{1}{f}\right)+\bar{N}(r, f)=S(r, f)$ and $\bar{N}\left(r, \frac{1}{g}\right)+\bar{N}(r, g)=S(r, g)$. If $f$ and $g$ share a finite complex number $a(\neq 0) I M$, then either $f \equiv g$ or $f . g \equiv a^{2}$.

Lemma 2.4 (see [5, Lemma 4]). If $f$ and $g$ share $(0,1),(1, \infty),(\infty, \infty)$ and $f \not \equiv g$ then

$$
\frac{f-1}{g-1}=e^{\alpha}
$$

and

$$
\frac{f}{g}=H
$$
where $\alpha$ is an entire function and $H$ is a meromorphic function with $\bar{N}\left(r, \frac{1}{H}\right)=$
$S(r)$ and $\bar{N}(r, H)=S(r)$, and $S(r)$ is the same as in Remark 1 .

Remark 3. Set

$$
H_{0}=\frac{e^{\alpha}}{H}
$$

Suppose that $H_{0}$ and $H$ are not constants. According to Lemma 2.4, we have

$$
T\left(r, \frac{H^{\prime}}{H}\right)+T\left(r, \frac{H_{0}^{\prime}}{H_{0}}\right)=S(r)
$$


It is clear from the assumptions of Lemma 2.4 that $H_{0}$ and $e^{\alpha}$ are not identically 1 , and from (2.2) and (2.3) we have

$$
f=\frac{e^{\alpha}-1}{H_{0}-1}, \quad g=\frac{e^{-\alpha}-1}{H_{0}^{-1}-1} .
$$

Lemma 2.5 (see [1, Proof of Theorem 1]). Suppose that $f$ and $g$ share $(0,1)$, $(1, \infty),(\infty, \infty)$, and suppose that $f$ is not any fractional linear transformation of $g$. Set

$$
h=\frac{\alpha^{\prime}}{H_{0}^{\prime} / H_{0}}=\frac{\alpha^{\prime}}{\alpha^{\prime}-H^{\prime} / H},
$$

where $H, H_{0}$ and $e^{\alpha}$ are given in Lemma 2.4 and Remark 3. Then

$$
-\frac{H_{0}^{\prime}}{H_{0}}(f-h)=\frac{g^{\prime}}{g} \frac{f-g}{g-1}
$$

and

$$
H_{0}^{\prime}=\frac{\alpha^{\prime}}{h} H_{0}
$$

Remark 4. Suppose that $f$ and $g$ satisfy the conditions of Lemma 2.5. If $f$ and $g$ are interchanged with one another, then we easily deduce from (2.2)(2.8) the following equation:

$$
\frac{H_{0}^{\prime}}{H_{0}}(g-h)=\frac{f^{\prime}}{f} \frac{g-f}{f-1} .
$$

Consequently, from (2.9)

$$
H_{0}^{\prime \prime}=\lambda H_{0}
$$

where

$$
\lambda=\left(\frac{\alpha^{\prime}}{h}\right)^{\prime}+\left(\frac{\alpha^{\prime}}{h}\right)^{2} .
$$

Of course, by (2.5) and (2.7), we deduce that $h$ is a small function of $f$ and so $g$, and hence that $\lambda$ is also a small function of $f$ and $g$.

Lemma 2.6 (see [5, Lemma 2]). If $f$ and $g$ share $(0,1),(1, \infty),(\infty, \infty)$ and suppose that $f \not \equiv g$, then $\bar{N}_{(2}\left(r, \frac{1}{f}\right)+N_{(2}\left(r, \frac{1}{f-1}\right)+N_{(2}(r, f)=S(r)$.

If in addition that $f$ and $g$ share $0 C M$, then $N_{(2}\left(r, \frac{1}{f}\right)=S(r)$.

LEMMA 2.7. Let $f$ and $g$ be two nonconstant meromorphic functions. If $a_{1}, a_{2}, a_{3}$ are three distinct small meromorphic functions of $g$ and $f \notin\left\{a_{1}, a_{2}, a_{3}\right\}$, then 


$$
T(r, f) \leq \bar{N}\left(r, \frac{1}{f-a_{1}}\right)+\bar{N}\left(r, \frac{1}{f-a_{2}}\right)+\bar{N}\left(r, \frac{1}{f-a_{3}}\right)+S(r, f)+S(r, g) .
$$

Proof. Set

$$
F(z)=\frac{f(z)-a_{1}(z)}{f(z)-a_{3}(z)} A(z),
$$

where $A(z)=\frac{a_{2}(z)-a_{3}(z)}{a_{2}(z)-a_{1}(z)}$.

Since $F=A\left\{1+\frac{a_{3}-a_{1}}{f-a_{3}}\right\}$, then

$$
T(r, F) \leq T(r, f)+S(r, g) .
$$

From (2.13) we have

$$
f=\frac{a_{3} B F-a_{1}}{B F-1},
$$

where $B=\frac{1}{A}$.

Suppose $a_{3} \equiv 0$. Then from (2.15), we get

$$
T(r, f) \leq T(r, F)+S(r, g),
$$

if $a_{3} \neq \equiv 0$, then $f=a_{3} \frac{F-\frac{a_{1}}{a_{3} B}}{F-\frac{1}{B}}$, from this we have (2.16).

We note that (2.14) and (2.16) imply that

$$
T(r, F)=T(r, f)+S(r, g) .
$$

By the second fundamental theorem of Nevanlinna

$$
\begin{aligned}
T(r, F) & \leq \bar{N}(r, F)+\bar{N}\left(r, \frac{1}{F}\right)+\bar{N}\left(r, \frac{1}{F-1}\right)+S(r, F) \\
& \leq \bar{N}\left(r, \frac{1}{f-a_{1}}\right)+\bar{N}\left(r, \frac{1}{f-a_{2}}\right)+\bar{N}\left(r, \frac{1}{f-a_{3}}\right)+S(r, f)+S(r, g),
\end{aligned}
$$

the lemma 2.7 follows from this inequality and (2.17).

\section{Proof of Theorem 1}

First we prove the first part of Theorem 1 , that $f$ is a fractional linear transformation of $g$.

Now, if $a=b$, by the assumptions of Theorem 1 and Gundersen's theorem (see [6, p. 262]), we get that $f$ and $g$ share $0,1, a, \infty \mathrm{CM}$, then from Theorem 
A, we deduce that $f$ is a fractional linear transformation of $g$. Therefore, it is enough to prove Theorem 1 when $a \neq b$.

We will assume that $f$ and $g$ share $(0,1),(1, \infty),(\infty, \infty)$ and $f$ is not any fractional linear transformation of $g$. It is clear from (2.2)-(2.4) that $\alpha, H$ and $H_{0}$ are not constants, otherwise, we get that $f$ is a fractional linear transformation of $g$. that

If $h \equiv c$, where $c$ is a nonzero constant, by integration (2.7), we find

$$
e^{((c-1) / c) \alpha}=A H
$$

where $A$ is a nonzero constant. From this equation and (2.3), we deduce that $f$ and $g$ share $0,1, \infty \mathrm{CM}$, it follows from Theorem $\mathrm{B}$ that $f$ is a fractional linear transformation of $g$. Therefore, we may suppose that

$$
h \neq \equiv, b \text {. }
$$

Let $z_{0}$ be a double zero of $g-b$, which is neither zero or pole of $\frac{H_{0}^{\prime}}{H_{0}}$, it follows from (2.8) and the assumptions of Theorem 1 that $f\left(z_{0}\right)=h\left(z_{0}\right)=a$, since $h$ is a small of $f$ and $g$ (see Remark 4) then by (2.5), (3.1) and by using (ii) of Lemma 2.1, we get

$$
N_{(2}\left(r, \frac{1}{g-b}\right) \leq N\left(r, \frac{1}{h-a}\right)+S(r)=S(r),
$$

here $S(r)$ is the same as in Remark 1. Similarly, we have

$$
N_{(2}\left(r, \frac{1}{f-a}\right) \leq N\left(r, \frac{1}{h-b}\right)+S(r)=S(r) .
$$

Hence,

$$
N_{(2}\left(r, \frac{1}{f-a}\right)+N_{(2}\left(r, \frac{1}{g-b}\right)=S(r) .
$$

By (2.6), we may define the following:

$$
\begin{gathered}
F_{a}=(f-a)\left(H_{0}-1\right)=e^{\alpha}-a H_{0}+a-1, \\
G_{b}=(g-b)\left(H_{0}^{-1}-1\right)=e^{-\alpha}-b H_{0}^{-1}+b-1, \\
\omega_{a}=\frac{F_{a}^{\prime}}{F_{a}} \quad \text { and } \quad \omega_{b}=\frac{G_{b}^{\prime}}{G_{b}} .
\end{gathered}
$$

It is clear from (iv) of Lemma 2.1, (2.5) and (3.2) that $F_{a}$ is not a constant. Moreover, if $\omega_{a}$ is a constant, then $F_{a}=A e^{z B}$, where $A$ and $B$ are nonzero constants. If $z_{0}$ is a simple zero of $f-a$, then $z_{0}$ is not a zero of $F_{a}$, from (3.3), $z_{0}$ must be a pole of $H_{0}$, again by (iv) of Lemma 2.1, (2.5) and (3.2), we obtain $T(r, f)=S(r, f)$, which is a contradiction. Therefore, $\omega_{a}$ is not a constant. Similarly, $\omega_{b}$ is not a constant. 
From Lemma 2.4 and (3.3), it is true that

$$
\bar{N}\left(r, F_{a}\right) \leq \bar{N}\left(r, H_{0}\right)+S(r)=S(r) .
$$

It follows from this inequality and (3.2)-(3.4), and by using (iv) of Lemma 2.1 that

$$
\begin{aligned}
T\left(r, \omega_{a}\right) & =N\left(r, \omega_{a}\right)+S(r) \\
& =\bar{N}\left(r, \frac{1}{F_{a}}\right)+S(r) \\
& =N\left(r, \frac{1}{f-a}\right)+N_{0}(r)+S(r) \\
& =T(r, f)+N_{0}(r)+S(r) .
\end{aligned}
$$

Let $z_{0}$ be a simple zero of $f-a$ such that $h\left(z_{0}\right) \neq 0, \infty$ and $\alpha^{\prime}\left(z_{0}\right) \neq 0$. Then from (2.2)-(2.4), we get $g\left(z_{0}\right)=b, e^{\alpha\left(z_{0}\right)}=\frac{a-1}{b-1}$ and $H_{0}\left(z_{0}\right)=\frac{b}{a} \frac{a-1}{b-1}$. By (3.3), (2.9), (2.11), (2.12) and by using the Taylor expansion of $F_{a}$ in a neighborhood of $z_{0}$, we get

$$
F_{a}=\tau_{1}\left(z_{0}\right)\left(z-z_{0}\right)+\tau_{2}\left(z_{0}\right)\left(z-z_{0}\right)^{2}+\tau_{3}\left(z_{0}\right)\left(z-z_{0}\right)^{3}+O\left(\left(z-z_{0}\right)^{4}\right)
$$

where

$$
\tau_{1}(z)=\left(\alpha^{\prime}-b \frac{H_{0}^{\prime}}{H_{0}}\right) \frac{a-1}{b-1}, \quad \tau_{2}(z)=\left(\left(\alpha^{\prime}\right)^{2}+\alpha^{\prime \prime}-b \frac{H_{0}^{\prime \prime}}{H_{0}^{\prime}} \frac{\alpha^{\prime}}{h}\right) \frac{a-1}{2(b-1)}
$$

and

$$
\begin{array}{r}
\tau_{3}(z)=\frac{1}{2} \frac{a-1}{b-1} \alpha^{\prime} \alpha^{\prime \prime}+\frac{a-1}{6(b-1)}\left(\alpha^{\prime}\right)^{3}+\frac{a-1}{6(b-1)} \alpha^{\prime \prime \prime}-\frac{a}{6}\left(\lambda^{\prime} \frac{b}{a} \frac{a-1}{b-1}+\lambda \frac{\alpha^{\prime}}{h} \frac{b}{a} \frac{a-1}{b-1}\right) . \\
\text { If } \tau_{1} \equiv 0 \text {, then } \frac{\alpha^{\prime}}{b}=\frac{H_{0}^{\prime}}{H_{0}} \text {, it is obvious from (2.7) that } h \equiv b \text {, which contradicts }
\end{array}
$$
to (3.1). Thus $\tau_{1} \not \equiv 0$. It seems from Lemma 2.4 that $\tau_{1}, \tau_{2}$ and $\tau_{3}$ are small meromorphic functions of $f$ and so $g$. By (3.4) and (3.6), and by using elementary techniques, we can easily show that

$$
\omega_{a}=\frac{1}{z-z_{0}}+\frac{B\left(z_{0}\right)}{2}+C\left(z_{0}\right)\left(z-z_{0}\right)+O\left(\left(z-z_{0}\right)^{2}\right),
$$

where $B=2 \frac{\tau_{2}}{\tau_{1}}$ and $C=2 \frac{\tau_{3}}{\tau_{1}}-\left(\frac{\tau_{2}}{\tau_{1}}\right)^{2}$.

Let us put

$$
H_{a}=\omega_{a}^{\prime}+\omega_{a}^{2}-B \omega_{a}-A,
$$

where $A=3 C-\frac{1}{4} B^{2}-B^{\prime}$. Evidently, $A, B$ and $C$ are small functions of $f$ and $g$. By using (3.7) and (3.8), it is not too difficult to verify that 


$$
H_{a}(z)=O\left(z-z_{0}\right) \text {. }
$$

Suppose $H_{a} \not \equiv 0$. Therefore, from (3.2) and (3.9), we see that

$$
N\left(r, \frac{1}{f-a}\right) \leq N\left(r, \frac{1}{H_{a}}\right)+S(r) \leq N\left(r, H_{a}\right)+S(r) .
$$

Of course, from (3.2), (3.3) and (iii) of Lemma 2.1

$$
N\left(r, \frac{1}{F_{a}}\right)=N_{1)}\left(r, \frac{1}{F_{a}}\right)+S(r) .
$$

Let $z_{*}$ be a simple zero of $F_{a}$. Therefore $\omega_{a}=\frac{1}{z-z_{*}}+C_{*}+O\left(z-z_{0}\right)$, where $C_{*}$ is a certain constant. Since $N_{(2}\left(r, \frac{1}{H_{0}-1}\right)=S(r)$. Then it is obvious from (3.2)-(3.4), (3.8), (3.10) and (3.11) that

$$
N\left(r, H_{a}\right)=\bar{N}\left(r, H_{a}\right)+S(r) .
$$

From the above, we can easily verify that possible poles of $H_{a}$ occur at zeros of $F_{a}$, as noted that if $z_{0}$ is a simple zero of $f-a$, which is neither zero of $h$ or $\alpha^{\prime}$, nor the pole of $h$, then $z_{0}$ must be a zero of $H_{a}$. Therefore the poles of $H_{a}$ occur at the zeros of $H_{0}-1$. It follows from (2.2)-(2.4) that the poles of $H_{a}$ occur at the zeros of $f-g$ but not zeros of $f(f-1)$ and $\frac{1}{f}$. Thus

$$
\bar{N}\left(r, H_{a}\right) \leq N_{0}(r)+S(r) .
$$

From this, (3.10), (3.12) and (iv) of Lemma 2.1, we get

$$
T(r, f) \leq N_{0}(r)+S(r) .
$$

Therefore, from this and (iii) of Lemma 2.1 that

$$
\bar{N}\left(r, \frac{1}{g^{\prime}}\right)=S(r)
$$
define

The same lines as the above, and by using (3.3) and (3.4), one can

$$
H_{b}=\omega_{b}^{\prime}+\omega_{b}^{2}-B_{1} \omega_{b}-A_{1},
$$

where $A_{1}$ and $B_{1}$ are small functions of $f$ and $g$. If $H_{b} \not \equiv 0$, by using the same techniques as the above, we obtain that

$$
T(r, g) \leq N_{0}(r)+S(r), \quad \text { and } \quad \bar{N}\left(r, \frac{1}{f^{\prime}}\right)=S(r) .
$$

Suppose that $H_{a} \not \equiv 0$ and $H_{b} \not \equiv 0$. We define the following:

$$
\varphi_{1}=\frac{f^{\prime \prime}}{f^{\prime}}-\frac{g^{\prime \prime}}{g^{\prime}}
$$


and

$$
\varphi_{2}=\left(\frac{f^{\prime \prime}}{f^{\prime}}-2 \frac{f^{\prime}}{f-1}\right)-\left(\frac{g^{\prime \prime}}{g^{\prime}}-2 \frac{g^{\prime}}{g-1}\right) .
$$

Since $f$ is not a fractional linear transformation of $g$, we get that $\varphi_{1}$ and $\varphi_{2}$ are not identically zeros, also we note that if $z_{0}$ is a simple pole of $f$ (or $z_{0}$ a simple zero of $f-1)$, then $z_{0}$ is also a zero of $\varphi_{1}\left(\right.$ or $\left.\varphi_{2}\right)$. Therefore, from the above and Lemma 2.6, we see that

$$
\begin{aligned}
N(r, f)+N\left(r, \frac{1}{f-1}\right) & \leq N\left(r, \frac{1}{\varphi_{1}}\right)+N\left(r, \frac{1}{\varphi_{2}}\right)+S(r) \\
& \leq N\left(r, \varphi_{1}\right)+N\left(r, \varphi_{2}\right)+S(r) \\
& \leq 2 \bar{N}\left(r, \frac{1}{f^{\prime}}\right)+2 \bar{N}\left(r, \frac{1}{g^{\prime}}\right)+S(r)=S(r) .
\end{aligned}
$$

From this and Lemma 2.3, we will reach a contradiction. Therefore, at least one of $H_{a}$ and $H_{b}$ is identically zero. Without loss of generality we will assume that $H_{a} \equiv 0$.

By (3.8) we have

$$
\omega_{a}^{\prime}=A+B \omega_{a}-\omega_{a}^{2} .
$$

From (3.3) and (3.4), we obtain

$$
F_{a}\left(\omega_{a}-\frac{H_{0}^{\prime}}{H_{0}}\right)=\left(\alpha^{\prime}-\frac{H_{0}^{\prime}}{H_{0}}\right)\left(e^{\alpha}-\zeta_{1}\right),
$$

where $\zeta_{1}=\frac{(a-1) H_{0}^{\prime}}{\alpha^{\prime} H_{0}-H_{0}^{\prime}}$. If $\omega_{a} \equiv \frac{H_{0}^{\prime}}{H_{0}}$, then from this, (2.5) and (3.5), we have $T(r, f)=S(r, f)$, which is impossible. Therefore, from (2.5), (3.3) and (3.14), we deduce $e^{\alpha} \not \equiv \zeta_{1}$. It follows from (3.11) and (3.14) that

$$
\begin{aligned}
\bar{N}\left(r, \frac{1}{\omega_{a}-\frac{H_{0}^{\prime}}{H_{0}}}\right) & \leq \bar{N}\left(r, \frac{1}{\alpha^{\prime}-\frac{H_{0}^{\prime}}{H_{0}}}\right)+\bar{N}\left(r, \frac{1}{e^{\alpha}-\zeta_{1}}\right)+\bar{N}\left(r, F_{a}\right)+S(r) \\
& =\bar{N}\left(r, \frac{1}{e^{\alpha}-\zeta_{1}}\right)+S(r) \\
& \leq \bar{N}\left(r, \frac{1}{F_{a}\left(\omega_{a}-\frac{H_{0}^{\prime}}{H_{0}}\right)}\right)+S(r)
\end{aligned}
$$




$$
\begin{aligned}
& \leq \bar{N}\left(r, \frac{1}{\omega_{a}-\frac{H_{0}^{\prime}}{H_{0}}}\right)+\bar{N}_{(2}\left(r, \frac{1}{F_{a}}\right)+S(r) \\
& =\bar{N}\left(r, \frac{1}{\omega_{0}-\frac{H_{0}^{\prime}}{H_{0}}}\right)+S(r),
\end{aligned}
$$

which gives

$$
\bar{N}\left(r, \frac{1}{\omega_{a}-\frac{H_{0}^{\prime}}{H_{0}}}\right)=\bar{N}\left(r, \frac{1}{e^{\alpha}-\zeta_{1}}\right)+S(r) \leq T\left(r, e^{\alpha}\right)+S(r) .
$$

Applying Lemma 2.7 to $a_{1}=0, a_{2}=\infty$ and $a_{3}=\zeta_{1}$, we get

$$
T\left(r, e^{\alpha}\right) \leq \bar{N}\left(r, \frac{1}{e^{\alpha}-\zeta_{1}}\right)+S(r) .
$$

This and the above inequality imply that

$$
\bar{N}\left(r, \frac{1}{\omega_{a}-\frac{H_{0}^{\prime}}{H_{0}}}\right)=\bar{N}\left(r, \frac{1}{e^{\alpha}-\zeta_{1}}\right)+S(r)=T\left(r, e^{\alpha}\right)+S(r) .
$$

By (2.2), we have

$$
\frac{f-g}{g-1}=e^{\alpha}-1
$$

From (3.16) we obtain

$$
T\left(r, e^{\alpha}\right)=\bar{N}\left(r, \frac{1}{e^{\alpha}-1}\right)+S(r)=N_{0}(r)+\bar{N}\left(r, \frac{1}{g}\right)+S(r) .
$$

From (3.15) and (3.17) we get

$$
\bar{N}\left(r, \frac{1}{\omega_{a}-\frac{H_{0}^{\prime}}{H_{0}}}\right)=N_{0}(r)+\bar{N}\left(r, \frac{1}{g}\right)+S(r) .
$$

Also, from (3.14), we see that 


$$
\begin{aligned}
& N\left(r, \frac{1}{\omega_{a}-\frac{H_{0}^{\prime}}{H_{0}}}\right)-N\left(r, \omega_{a}-\frac{H_{0}^{\prime}}{H_{0}}\right)+S(r) \\
& \quad=N\left(r, F_{a}\right)+N\left(r, \frac{1}{e^{\alpha}-\zeta_{1}}\right)-N\left(r, \frac{1}{F_{a}}\right)-N\left(r, e^{\alpha}-\zeta_{1}\right) .
\end{aligned}
$$

From this, (2.5), (3.3)-(3.5), (3.11) and Lemma 2.4, we get

$$
N\left(r, \frac{1}{\omega_{a}-\frac{H_{0}^{\prime}}{H_{0}}}\right)=N\left(r, H_{0}\right)+N\left(r, \frac{1}{e^{\alpha}-\zeta_{1}}\right)+S(r) .
$$

Since

$$
N\left(r, \frac{1}{e^{\alpha}-\zeta_{1}}\right) \leq T\left(r, e^{\alpha}\right)+S(r)
$$

therefore, from (2.4), (3.15), (3.17), (3.19) and (3.20), we obtain

$$
N\left(r, \frac{1}{\omega_{a}-\frac{H_{0}^{\prime}}{H_{0}}}\right)=N\left(r, \frac{1}{H}\right)+N_{0}(r)+\bar{N}\left(r, \frac{1}{g}\right)+S(r) .
$$

From (3.3) and (3.4), we have

$$
F_{a}\left(\omega_{a}-\alpha^{\prime}\right)=a\left(\alpha^{\prime}-\frac{H_{0}^{\prime}}{H_{0}}\right)\left\{H_{0}-\frac{\alpha^{\prime}(a-1)}{\zeta_{2}}\right\},
$$

where $\zeta_{2}=a\left(\alpha^{\prime}-\frac{H_{0}^{\prime}}{H_{0}}\right)$.

If $\omega_{a} \equiv \alpha^{\prime}$, then from (3.4), we have $F_{a}=A e^{\alpha}$, where $A$ is a nonzero constant, from this, (3.2), (3.3) and Lemma 2.4, we get $N\left(r, \frac{1}{f-a}\right)=S(r)$, which contradicts to (iv) of Lemma 2.1. Therefore, $\omega_{a} \not \equiv \alpha^{\prime}$, that is $H_{0} \not \equiv$ $\frac{\alpha^{\prime}(a-1)}{\zeta_{2}}$.

In the same manner as the above and by using (3.22), we can get

$$
N\left(r, \frac{1}{\omega_{a}-\alpha^{\prime}}\right)=\bar{N}\left(r, \frac{1}{\omega_{a}-\alpha^{\prime}}\right)+S(r)=N_{0}(r)+\bar{N}(r, g)+S(r) .
$$

Similarly, we can obtain the following

$$
N\left(r, \frac{1}{\omega_{a}}\right)=\bar{N}\left(r, \frac{1}{\omega_{a}}\right)+S(r)=N_{0}(r)+\bar{N}\left(r, \frac{1}{g-1}\right)+S(r) .
$$


Assume that the zero is a solution of the differential equation (3.13). Since $T\left(r, \omega_{a}\right)=O(T(r, f))$, then by using (ii) of Lemma 2.2, we obtain that

$$
\bar{N}\left(r, \frac{1}{\omega_{a}}\right)=S(r) \text {. }
$$

We see that (2.3) implies that $\frac{f-g}{g}=H-1$, and hence that

$$
\bar{N}\left(r, \frac{1}{H-1}\right)=N_{0}(r)+\bar{N}\left(r, \frac{1}{g-1}\right)=S(r),
$$

by (3.24) and (3.25). From Lemma 2.4 and by applying Lemma 2.7, we get $T(r, H)=S(r)$. However, if $z_{0}$ be a simple zero of $f-a$, then $z_{0}$ is a zero of $g-b$, which implies that $z_{0}$ is also a zero of $H-\frac{a}{b}$, by (2.3). Since $f$ is not any fractional linear transformation of $g$, then $H \neq \equiv \frac{a}{b}$, it follows from this and (3.2)
that

$$
N\left(r, \frac{1}{f-a}\right) \leq N\left(r, \frac{1}{H-\frac{a}{b}}\right) \leq T(r, H)+S(r)=S(r, f),
$$

which is a contradiction with (iv) of Lemma 2.1. This proves that the zero is not a solution of (3.13). It follows from (iii) of Lemma 2.2, (3.24) that

$$
T\left(r, \omega_{a}\right)=N\left(r, \frac{1}{\omega_{a}}\right)+S(r)=N_{0}(r)+\bar{N}\left(r, \frac{1}{f-1}\right)+S(r) .
$$

Assume that $\alpha^{\prime}$ is a solution of the differential equation (3.13). By (ii) of Lemma 2.2 and (3.23), we get

$$
N_{0}(r)+\bar{N}(r, g)=S(r)
$$

From (2.4), we see that $H_{0}-1=\frac{f-g}{f(g-1)}$, this and (3.27) yield

$$
\bar{N}\left(r, \frac{1}{H_{0}-1}\right)=N_{0}(r)+\bar{N}(r, g)+S(r)=S(r) .
$$

Therefore, from this, Lemma 2.4 and applying Lemma 2.7, we get

$$
T\left(r, H_{0}\right)=S(r) .
$$

If $z_{0}$ be a simple zero of $f-a$, then $z_{0}$ is a zero of $g-b$, which implies that $z_{0}$ is also a zero of $H_{0}-\frac{a-1}{b-1} \frac{b}{a}$, by (2.4). Since $f$ is not any fractional linear transformation of $g$, then $H_{0} \not \equiv \frac{a-1}{b-1} \frac{b}{a}$, it follows from this and (3.2) that 


$$
N\left(r, \frac{1}{f-a}\right) \leq N\left(r, \frac{1}{H_{0}-\frac{a-1}{b-1} \frac{b}{a}}\right) \leq T\left(r, H_{0}\right)+S(r)=S(r, f),
$$

which is a contradiction with (iv) of Lemma 2.1. This proves that $\alpha^{\prime}$ is not a solution of (3.13). It follows from (iii) of Lemma 2.2, (3.23) that

$$
T\left(r, \omega_{a}\right)=N\left(r, \frac{1}{\omega_{a}-\alpha^{\prime}}\right)+S(r)=N_{0}(r)+\bar{N}(r, f)+S(r)
$$

Similarly, if $\frac{H_{0}^{\prime}}{H_{0}}$ is a solution of (3.13), then from (2.2), (3.18) and by using Lemma 2.7, we find that $T\left(r, e^{\alpha}\right)=S(r)$, from this, (2.2) and (3.2), we conclude $N\left(r, \frac{1}{f-a}\right)=S(r)$, which is a contradiction with (iv) of Lemma 2.1. Therefore, $\frac{H_{0}^{\prime}}{H_{0}}$ is not a solution of (3.13). It follows from (iii) of Lemma 2.2 and (3.21)

$$
T\left(r, \omega_{a}\right)=N\left(r, \frac{1}{\omega_{a}-\frac{H_{0}^{\prime}}{H_{0}}}\right)+S(r)=N\left(r, \frac{1}{H}\right)+N_{0}(r)+\bar{N}\left(r, \frac{1}{f}\right)+S(r),
$$

From (3.5), (3.26), (3.29) and (3.30) we obtain

$$
\begin{gathered}
T(r, f)=N\left(r, \frac{1}{H}\right)+\bar{N}\left(r, \frac{1}{f}\right)+S(r), \\
T(r, f)=\bar{N}(r, f)+S(r)
\end{gathered}
$$

and

$$
T(r, f)=\bar{N}\left(r, \frac{1}{f-1}\right)+S(r) .
$$

It follows from (3.31)-(3.33)

$$
3 T(r, f)=N\left(r, \frac{1}{H}\right)+\bar{N}\left(r, \frac{1}{f}\right)+\bar{N}(r, f)+\bar{N}\left(r, \frac{1}{f-1}\right)+S(r) .
$$

It is clear from (3.34) and (v) of Lemma 2.1 that

$$
2 T(r, f)+N_{0}(r)=T(r, g)+N\left(r, \frac{1}{H}\right)+S(r) .
$$

Assume that $H_{b} \not \equiv 0$. Then, we have

$$
T(r, g) \leq N_{0}(r)+S(r), \quad \text { and } \quad \bar{N}\left(r, \frac{1}{f^{\prime}}\right)=S(r) .
$$


From this we obtain

$$
T(r, g)=N_{0}(r)+S(r),
$$

by using (iii) of lemma 2.1. Substituting this into (3.35), we get $2 T(r, f)=$ $N\left(r, \frac{1}{H}\right)+S(r)$, from this and (3.31), we immediately obtain $T(r, f)=S(r)$, which is impossible. This shows that $H_{b} \equiv 0$. In the same manner as the proof (3.32), we can obtain $T(r, g)=\bar{N}(r, g)+S(r)$, from this and (3.32), we get $T(r, g)=T(r, f)+S(r)$. From this, (3.31) and (3.35) we have

$$
N_{0}(r)+\bar{N}\left(r, \frac{1}{f}\right)=S(r) .
$$

This and (2.2) yield that $T\left(r, e^{\alpha}\right)=S(r)$. If $z_{0}$ be a simple zero of $f-a$, then $z_{0}$ is a zero of $g-b$, which implies that $z_{0}$ is also a zero of $e^{\alpha}=\frac{a-1}{b-1}$, by using (2.2). So that from this and (3.2) imply that

$$
N\left(r, \frac{1}{f-a}\right) \leq N\left(r, \frac{1}{e^{\alpha}-\frac{a-1}{b-1}}\right)+S(r) \leq T\left(r, e^{\alpha}\right)+S(r)=S(r),
$$

this is a contradiction with (iv) of Lemma 2.1. This proves the first part of Theorem 1, when $f$ and $g$ share $(0,1),(1, \infty),(\infty, \infty)$.

Now if $f$ and $g$ share $(0, \infty),(1,1),(\infty, \infty)$ (or $(\infty, 1),(0, \infty),(1, \infty)$ ), then $F=1-f$ and $G=1-g$ (or $F=1 / f$ and $G=1 / g$ ) share $(0,1),(1, \infty),(\infty, \infty)$, and $F=1-a \Leftrightarrow G=1-b$ (or $F=\frac{1}{a} \Leftrightarrow G=\frac{1}{a}$ ), from the last part of the above proof, we have that $F$ is a fractional linear transformation of $G$, which leads that $f$ is a fractional linear transformation of $g$. This proves the first part of Theorem 1 .

In order to prove the second part of Theorem 1, we assume that $f$ is a fractional linear transformation of $g$. Therefore

$$
f=\frac{A g+B}{C g+D}
$$

where $A, B, C$ and $D$ are constants and $A D-B C \neq 0$. We see $A B C D=0$, otherwise, we have three distinct values of Picard exceptional of $g$. CASE 1. Suppose that $A=0$. Then $D=0$ (otherwise, $0, \infty,-\frac{D}{C}$ are Picard
exceptional values of $g$ ). So that $f . g \equiv 1$, which is (ii).

CASE 2. Suppose that $C=0 . \quad$ Assume that $B=0$, then $f=\frac{A}{D} g . \quad$ If $\frac{A}{D}=1$, then we deduce (i). If $\frac{A}{D} \neq 1$, then 1 and $\frac{A}{D}$ are Picard exceptional values of 
$f$. If $\frac{A}{D}=a$, then we have (v), otherwise $\frac{A}{D}=\frac{a}{b}$, which is (iii). Assume that $B \neq 0$, then from (3.36), we have $f=\frac{A}{D} g+\frac{B}{D}$. If $\frac{B}{D}=1$, then $f=\frac{A}{D} g+1$, and hence that 0 and 1 are Picard exceptional values of $f$ and $g$, so that $\frac{D}{A}=-1$, which implies (iv). If $\frac{B}{D} \neq 1$, then 0 and $\frac{B}{D}$ are Picard exceptional values of $f$, and 0 and $-\frac{B}{A}$ are Picard exceptional values of $g$. If $\frac{B}{D}=a$, then $f=\frac{A}{D} g+a$, since 1 is not a Picard exceptional value of $f$ and $g$ then $\frac{A}{D}=1-a$, which is (vi). If $\frac{B}{D} \neq a$, hence that there are $z_{0}$ and $z_{1}$ such that $f\left(z_{0}\right)=a, g\left(z_{0}\right)=b$ and $f\left(z_{1}\right)=g\left(z_{1}\right)=1$, from these, we have (vii).

CASE 3. Suppose that $D=0$. If $A \neq 0$, then $A B C \neq 0$, and we have $0, \infty$, $-\frac{B}{A}$ are Picard exceptional of $g$, which is impossible. Thus, $A=0$, and hence we will deduce (ii).

CASE 4. Suppose that $B=0$ and $A C D \neq 0$. Therefore, (3.36) gives

$$
f=\frac{x g}{g-y}
$$

where $x=A / C, y=-D / C$. If $x=1$, then $\infty$ and 1 are Picard exceptional values of $f$ and $g$, so that $y=1$, which implies the case $(x)$ in Theorem 1 . Suppose that $x \neq 1$. Then from (3.37), 1 is not a Picard exceptional value of $f$ and $g$. Therefore, by (3.37) we get

$$
x+y=1 .
$$

If $x=a$, then from (3.37) and (3.38), we get (viii). If $x \neq a$, then $a$ is not a Picard exceptional value of $f$, so that there is $z_{0}$ such that $f\left(z_{0}\right)=a, g\left(z_{0}\right)=b$, it follows from (3.37) that $b x+a y=a b$, from this and (3.38), we get (ix). This proves Theorem 1 .

Acknowledgments. Research of the first author supported by State Post Doctoral Fellowship Program (Grant LRB05-013). The first author is grateful to Professor Hong You for his warm support and help at Harbin Institute of Technology. The authors would like to thank the referee for many useful suggestions and comments.

\section{REFERENCES}

[ 1 ] T. C. Alzahary AND H. X. YI, Weighted sharing three values and uniqueness of meromorphic functions, J. Math. Anal. Appl. 259 (2004), 247-257. 
[2] G. Brosch, Eindeutigkeitssätze für Meromorphe Funktionen, Thesis, Techincal University of Aachen, 1989.

[ 3 ] W. K. Hayman, Meromorphic functions, Clarendon Press, Oxford, 1964.

[4] G. Gundersen, Meromorphic functions that share three or four values, J. London Math. Soc. (2) 20 (1979), 457-466.

[5] I. LAHIRI, Weighted sharing of three values and uniqueness of meromorphic functions, Kodai Math. J. 24 (2001), 421-435.

[6] H. X. YI AND C. C. YANG, Uniqueness theory of meromorphic functions, Science Press, Beijing, 1995.

Department of Mathematics

HaRbin Institute of TeChNOlogy

Harbin, Heilonguiang 150001

People's Republic of China

E-mail: thammra@yahoo.com

CuRrent ADDRESS:

Department of Mathematics

HARBIN ENGINEERING UNIVERSITY

Harbin, HeilongJiang 150001

People's Republic of China

Department of Mathematics

SHANDONG UNIVERSITY

Jinan, Shandong 250100

People's Republic of China

E-mail: hxyi@sdu.edu.cn 